\title{
Different Questions Beg Different Methods
}

A s a recent historical review demonstrated, the Society of General Internal Medicine (SGIM) arose some 25 years ago out of a collective sense among internists that medical specialization was narrowing clinical perspectives and that existing research was neither asking nor answering the important questions in medical education and primary care. ${ }^{1}$ To study complex clinical phenomenon more adequately, many SGIM members have taken the lead in developing innovative research methods and approaches, largely in the areas of clinical epidemiology and health services research. These quantitative techniques have helped make clinical research more rigorous and relevant and have led to the nonstatistical "p values" vital to academic researchers: publications, promotions, and prestige. However, the nearexclusive focus on quantitative measurement and statistical abstraction has also contributed to a turning away from the early holistic and patient-centered goals of general internal medicine.

Despite these trends, a few clinicians and researchers have used methods drawn from fields such as anthropology, sociology, and philosophy to research questions not easily studied by quantitative techniques. Although SGIM meetings have recently sponsored discussions of qualitative research, and instructional books on qualitative methods have been published for clinical audiences, qualitative research has hardly found a place in the mainstream medical literature. Most clinicians might reasonably still ask: what exactly is qualitative research and what can it do for general medicine?

In most contexts, the term qualitative research has been used to denote research methods that do not involve manipulation of numbers beyond simple descriptive statistics. If that were all that researchers meant, it might be more accurate to speak of "nonquantitative" research. Using such a negative definition, we would at least honestly acknowledge the less privileged status of the methods and researchers subsumed under the heading. The negative definition would also avoid lumping together methods as widely divergent as focus groups and historical analysis.

On the other hand, the word "qualitative" has a more positive and an accurate sense when used to describe novel research methods in primary care. A dictionary definition of quality is the "peculiar and essential character" of things. ${ }^{2}$ Qualitative analysis in chemistry, for example, aims to identify the component elements in a solution or mixture. As a goal, qualitative primary care research aims to identify the essential component parts of clinical phenomena, recognizing that traditional quantitative methods often fail to provide a comprehensive understanding. Like qualitative analysis in chemistry, qualitative methods can identify the fundamental units that should be understood prior to quantitative manipulation.

To exemplify this "essential" meaning of qualitative analysis, consider the extensive literature on cardiovascular risk factors. While epidemiologic trials such as the Framingham study have greatly advanced our knowledge, they have not by themselves identified which factors are essential or fundamental. The problem is that concepts about what is essential or fundamental too often are equated with statistical concepts such as independence. Obesity may fail as an independent risk factor for coronary heart disease in regression analysis, but it may nevertheless be an essential factor. Getting fat may be the marker that identifies many other "independent" risks and may be the most visible way individuals monitor their cardiovascular risk. The risk factor categorization problem may necessitate something like qualitative analysis in chemistry. Such an analysis might lead to a better understanding of which aspects of individual and group behavior and physiology "hang together" and which are amenable to population interventions, individual behavior change, or physiologic manipulation. Such qualitative analysis might include open-ended interviews with small groups of high risk individuals or it might include a sociohistorical analysis of how certain risk factor concepts became so popular within medicine, while others were dismissed.

The report in this issue of the Journal by Shimada and colleagues, describing factors that determine medical noncompliance among Cambodian refugees in Seattle, illustrates three additional features of qualitative research. ${ }^{3}$ First, investigators often determine methods and variables in the process of inquiry rather than rigidly applying a predetermined set of rules. Shimada analyzed transcripts of open structured interviews and then determined a taxonomy of reasons for patient noncompliance. In this study, subjects helped interviewers understand what was important, leading to further questions and new analyses.

Second, investigators carrying out a qualitative study are often an inextricable part of the phenomena being investigated. Their perspective may be crucial to the interpretation of research findings. In the Cambodian study, for example, the investigators' literal translation of "strong medicine" into English did not immediately reveal its meaning. We have no concept that exactly matches the Cambodian idea. The investigators needed to reconcile this new idea with their own views of how the world works. Their own perspective was an important part of this process. This "bias" is not something to be eliminated, but is a productive element, a foundation for formulating questions and understanding answers in the process of the research. 
Third, investigators doing qualitative research generally make sense of their data, which typically appears as written, spoken, cultural, or behavior expressions, in light of larger linguistic, historical, and cultural contexts. Shimada analyzed medical noncompliance in light of the native beliefs of their subjects. ${ }^{3}$ Such an analysis required sociocultural, not statistical, inference. Put an other way, this form of inquiry focused on meanings more than mechanisms. As a result, investigators reframed the concept of medical noncompliance in a less pejorative and normative way. They learned about patients' beliefs and behaviors without the distorting assumption that their job was to explain deviance from the right way of believing and acting.

Why should such methods matter to internists? First, qualitative inquiry is especially suited to areas of primary care that have both social and clinical dimensions, such as medical ethics, domestic and societal violence, cross-cultural medicine, and substance abuse. Too often the limits to using clinical epidemiologic methods in these areas are in principle acknowledged but in practice forgotten. Clinical epidemiologic methods might help identify risk factors for women subject to domestic abuse, but close observation and in-depth interviews with abused women might yield a more comprehensive understanding of why battered women avoid medical care. Similarly, a controlled trial might test whether an ethics curriculum for houseofficers increases the number of completed advanced directives on a medical service, while focus groups might be a better way to understand their ethical reasoning. Simply put, different questions beg different methods.

For the academic researcher, qualitative inquiry can also clarify the role, the limits, and the implicit assumptions of quantitative research. P-values and confidence intervals may tell us what findings are significant and to what degree, but often they do not tell us whether results answer appropriate and worthwhile questions. An exclusive focus on what is easily measurable may silently preempt a broad range of alternative questions and reduce many dimensions of clinical experience to a single and misleading numerical dimension. For example, cardiovascular risk factor equations require that putative factors operate at the level of the individual. While pack years of smoking can be entered into such equations, the role of tobacco advertising or price subsidies cannot. The influence of such nonspecific factors - which might be uncovered and delineated by cross-cultural or historical studies - is no less important because they cannot easily be modeled in mathematical equations.

Finally, in many situations, qualitative methods can be used prior to clinical epidemiologic study to determine the most relevant factors to measure. The authors of the Cambodian study, for example, having discovered the cultural component to medical noncompliance among their patients, might now recruit standard clinical epidemiologic methods to study the effects of physician education, phone visits, or culturally trained visiting nurses on medical noncompliance. By generating alternative questions and conclusions, qualitative inquiry can help investigators better focus their questions and methods. Qualitative inquiry can thus defend against what has been called a type III statistical error: giving a precise and significant statistical answer to the wrong question. $^{4}$

Philosopher Hans-Georg Gadamer has written that "no productive scientist can really doubt that methodical purity is indispensable in science; but what constitutes the essence of research is much less merely applying the usual methods than discovering new ones-and underlying that, the creative imagination of the scientist."5 For issues and questions largely inaccessible to quantitative methods, JGIM will consider manuscripts that creatively utilize ideas and methods common to nonclinical disciplines, including (but not limited to) sociology, psychology, anthropology, linguistics, history, critical theory, cultural studies, and philosophy. We will favor manuscripts that apply discrete qualitative methods to specific questions, but we will consider interpretive pieces that shed new light on well-worn subjects. Whatever their form, manuscripts must be written for physicians unfamiliar with these fields and address the concerns of general internists. By reaching out to new approaches, JGIM looks to revitalize the "essence of research" within its pages. - Michael Berkwis, MD, Robert Wood Johnson Clinical Scholars Program, University of Pennsylvania, Philadelphia, PA; and Robert Aronowitz, MD, University of Medicine and Denistry of New Jersey/ Robert Wood Johnson Medical School, Camden, $\mathrm{NJ}$ 08103

\section{REFERENCES}

1. Noble J, Goldman L, Marvinney SL, Dale DC. The Society of General Internal Medicine from Conception to Maturity: 1970s to 1994. J Gen Intern Med. 1994:9(Aug suppl):S1-S44

2. Webster's New Collegiate Dictionary. Springfield MA: G. and C. Merriam Company, 1973:944.

3. Shimada J, Jackson JC, Goldstein E, Buchwald D. "Strong medicine": Cambodian views of medicine and medical compliance. J Gen Intern Med. 1995;10:369-74

4. Kimball JW. Errors of the third kind in statistical consulting. J Am Stat Assoc. 1957;52:133-42.

5. Gadamer HG. Afterword. In: Truth and Method. 2nd ed. New York: Crossroad Publishing, 1989:551-2. 